\title{
Morphological and Structural Properties of Porous Silicon (PSi)
}

\author{
Ayad Jumaah Kadhim ${ }^{1}$, Muneer H. Jaduaa Alzubaidy ${ }^{1}$, Ahmed N. Abd² \\ ${ }^{1}$ College of Science, Wasit University, Wasit, Iraq \\ 2Department of Physics, Faculty of Science, University of Al-Mustansiriyah, Baghdad, Iraq \\ *ahmed_naji_abd@uomustansiriyah.edu.iq
}

Keywords: porous silicon, $\mathrm{XRD}$, average grain size, AFM

\begin{abstract}
This study includes the effect of the etching time on the morphology of the surfaces produced using the electrochemical method of silicon( $p$-type), where it was found that the etching leads to increase the porosity layer of silicon. The production of nanocrystalline structures and control of their production conditions is the first step to control the properties of the devices. These applications are very important for the etching of renewable energy.
\end{abstract}

\section{Introduction}

The idea of silicon planking has become very important in the science of semiconductors and solar cells because it has a great effect on the improvement and production of devices with wide uses in various joints of life. The etching work has reached the so-called nanotechnology as the particles that are less than One hundred nanometers (a nanometer part of a thousand million meters) gives the substance to be incorporated into new properties [1]. As the size of the material approached the atomic dimensions, the subject was subjected to the laws of quantum mechanics instead of the laws of classical physics [2]. The adoption of the behavior of the material on its size enables us to control the engineering of its properties. There are two ways to manufacture a nanoscale size of the material, one from top to bottom and the other from bottom to top [3, 4]. Silicon strips can be used as separators and reagents and may be used to produce solar cells inducted with materials that increase their efficiency or even increase the surface area of the solar cell exposed to light. This will encourage the entry of such research Theoretical

\section{Surface Morphology}

The study of the surface morphology of the material is of importance that will explain the properties and behavior of the material as nanomaterials are different from other traditional materials by increasing the surface area and the presence of most of their atoms on those surfaces because all activities and chemical and physical changes always occur on the surface [3]. The study of morphology shows how the distribution and arrangement of atoms on the surface and explain the difference in homogeneity and properties related to each crystal structure [5]. Surface morphology is normally influenced by the technique used and the preparation conditions. The morphology of the surface of the slide prepared by electrochemical etching using the atomic force microscope (AFM) showed that the surface had a nanoscale structure and was highly homogenous.

\section{Nanoporous Silicon}

The discovery of porous silicon gave a new dimension to silicon-based techniques in 1990 and the discovery of photosynthesis at room temperature of porous silicon by (Kanham) [6]. Non-use the Bulk Silicon in the field of light emitting diode (LED), because silicon is a semi-conductive material with an indirect power gap, the optical luminescence occurs at room temperature very small $[6,7]$.

Porous silicon is new material of great practical and theoretical importance due to its quantitative properties. Nanoparticle silicon is prepared in a number of ways, including photochemical crystallization of a crystalline silicon chip surface in a hydrolyzed hydrofluoride solution. The research is now intensively focused on studying the properties of nanocrystalline silicon as a glowing material. Nanoparticle silicon is not limited to photovoltaic applications, on its properties 
as a crystal with a bright light [8]. Which change the optical properties and absorption, making them sensitive to specific wavelengths and useful in optical applications. By controlling the etching conditions (time, slide specification, acid concentration) so that a suitable crystalline structure of porous silicon nanoparticles can be obtained with different porous layers. The large surface area of the nanoparticle silicon and its unique surface structure provided ideal conditions for its use as a light sensor or diode. The difference between the number of carriers on the surface and at the base [3]. And that the importance of the use of nanoparticle silicon in the manufacture of photodiode and diodes and study of their electrical properties come in particular from the industrial point of view compared to other materials used in this area of low cost of manufacture and the possibility of preparing the surface for high selectivity [9]. As well as the provision of raw material and non-loss of energy due to the small size of manufacturing.

\section{Nanostructure porous silicon}

The bulk silicon is an unsuitable material for photovoltaic applications because of the occurrence of many non-radioactive phenomena that hinder the process of radio pairing. One possible way to increase the phenomenon of quantitative restriction is to limit the movement of charge carriers in the crystalline vacuum, thereby reducing the probability of non-radioactive phenomena, If we can limit the movement of cargo carriers in a very small area, far from the nanometer, and it is well known that the properties of the material in such a region vary greatly. Since the De Broglie wavelength of an electron or gap is $1 \mathrm{~nm}$, the particles (electrons and holes) in this region behave like particles in a box and can be solved by quantum mechanics. This is called the process of inventory of electrons in a region with nanomaterials in the sense of quantitative restriction, called the physical crystalline structure in which carriers are limited to the quantum of nanostructured material. Depending on the shape of the crystalline nanoparticle, the quantitative enumeration process can occur in only one dimension or in two or three dimensions [10]. Each direction of the inventory corresponds to a change in the wave of the particle and as a result a series of discrete levels appear. Bearing in mind that the charge carrier is free of movement in all directions in crystal size, the two-dimensional (2-D) structure can move freely only in two directions while the third direction determines the direction of quantitative restriction. In the one-dimensional (1-D) structure there is only one possible direction for free movement and two possible directions for quantification can occur. In the zero (0-D) structure it gets completely quantified in all directions and the particle cannot move free movement at all. The nanotube structure (2-D) is called the quantum well (a thin membrane) and is obtained by depositing layers of different materials with nanometer dimensions. The structure (1-D) is called the quantum wire and is obtained by specific processes. The structure $(0-\mathrm{D})$ is called the quantitative points and is prepared in different ways such as chemical vapor deposition, ionization, etching photochemical method [11]. Porous silicon is one of the most important examples of nanotubes from quantitative points and can be formed in a binary or three-dimensional structure. Fig. 1 is a schematic representation of the different nanoscale models [12]. 
$0-\mathrm{D}$

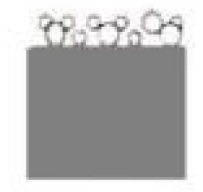

Nanocrystals by deposition

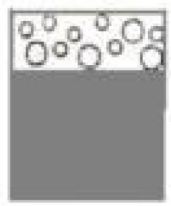

Nanocrystals

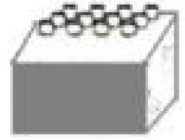

Quantum dots

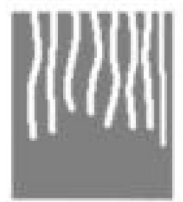

Porous Si
$1-\mathrm{D}$
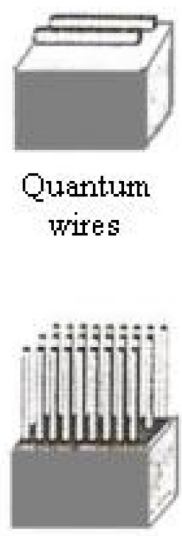

Pillars
2-D
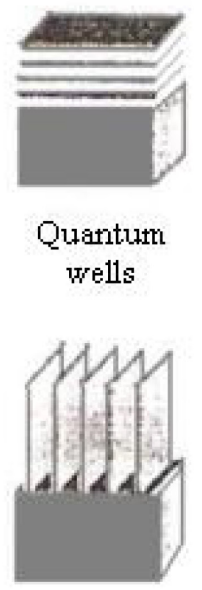

Tralls

Figure 1. A schematic representation of the models of nanoparticle structures [12]

\section{Methods for preparation of Nanoporous silicon}

There are several methods to prepare the porous silicon and the properties of this silicon vary from one method to another. The following are the most common methods of preparing porous silicon: Photochemical etching, Photoelectrochemical etching, Stain etching, Laser etching, Electrochemical etching. The method used in this research is the electrochemical method.

\section{Electrochemical etching}

The method of electrochemical etching was chosen in this research because it is distinguished from the other by controlling the properties of the produced porous silicon, producing large surface areas and homogenizing the voltages being controlled on the models and vertically, which leads to the production of relatively similar nanostructures during the process of drilling there two atoms of hydrogen are aligned with the silicon atom and the ratios of hydrogen atoms are reduced when they reach the electronic polishing system at the surface and disappear during this process. The semispherical reaction of the anode during the hole formation process is written in the following formula:

$$
\mathrm{Si}+6 \mathrm{HF} \rightarrow \mathrm{H}_{2} \mathrm{SiF}_{6}+\mathrm{H}_{2}+2 \mathrm{H}^{+}+2 \mathrm{e}^{-}
$$

During the process of electronic refinement, the formula is written in this format:

$$
\mathrm{Si}+6 \mathrm{HF} \rightarrow \mathrm{H}_{2} \mathrm{SiF}_{6}+4 \mathrm{H}^{+}+4 \mathrm{e}^{-}
$$

The final and constant output of the presence of silicon inside (HF) and its interaction together produce $\mathrm{H}_{2} \mathrm{SiF}_{6}$ or be some of his ions. This means that during the process of forming holes two of the four electrons on the silicon atom leave the atom and move with the removed part while the other two remain attached to the silicon atom and suffer erosion and emancipation later. Lehmann and Gosele [14] are suggested the most acceptable mechanical disintegration of Fig. 2. 

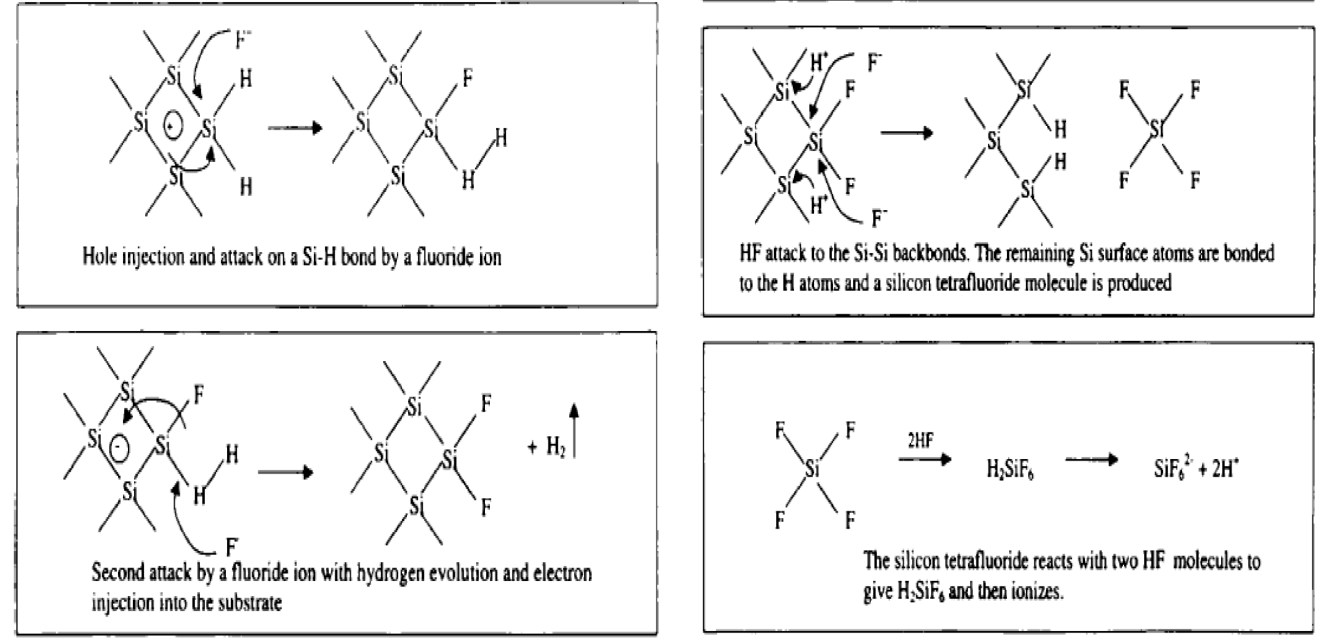

Figure 2. Proposed silicon decomposition scheme by Lehmann and Gosele [13]. Experimental part

\section{X-ray diffraction}

The X-ray diffraction spectra showed a clear difference between the surface of the pure silicon (c-Si) and the porous silicon surface (p-pSi), which is during the period of etching at $10 \mathrm{~min}$ and $10 \mathrm{~mA} / \mathrm{cm}^{2}$. A single strong, sharp and narrow peak was observed at $2=69.7^{\circ}$ on (400) confirming the PSi monomer structure. The tests showed that PSi had a crystalline glomerular structure in accordance with international standard (JCPDS 2011, ICDD1997). Fig. 3 shows XRD diffraction of pure (c-Si) and (p-PSi), as well as X-ray of pure silicon (c-Si). The figure shows that the pure silicon peak has a high value compared to $\mathrm{p}$-PSi. This result is due to the deviation of the rays from the crystals between the pores. According to this diagram, we can confirm that the p-PSi layer remains crystallize.

Table 1. The most important structural parameters of x-ray of (p-PSi)

\begin{tabular}{|c|c|c|c|c|c|c|}
\hline $\begin{array}{c}\text { Etching } \\
\text { time } \\
(\mathrm{min})\end{array}$ & $2 \theta(\mathrm{deg})$ & $\mathrm{d}(\mathrm{A})$ & $\begin{array}{c}\text { FWHM } \\
(\mathrm{deg})\end{array}$ & $\mathrm{D}(\mathrm{nm})$ & $\begin{array}{c}\text { Lattice } \\
\text { Constant } \\
(\mathrm{nm})\end{array}$ & $\begin{array}{c}\text { Strain*10 }^{-3} \\
\text { Lines }^{-2} \mathrm{~m}^{-4}\end{array}$ \\
\hline 10 & 69.96 & 1.348 & 0.13 & 74.421 & 1.348 & 27.88 \\
\hline
\end{tabular}

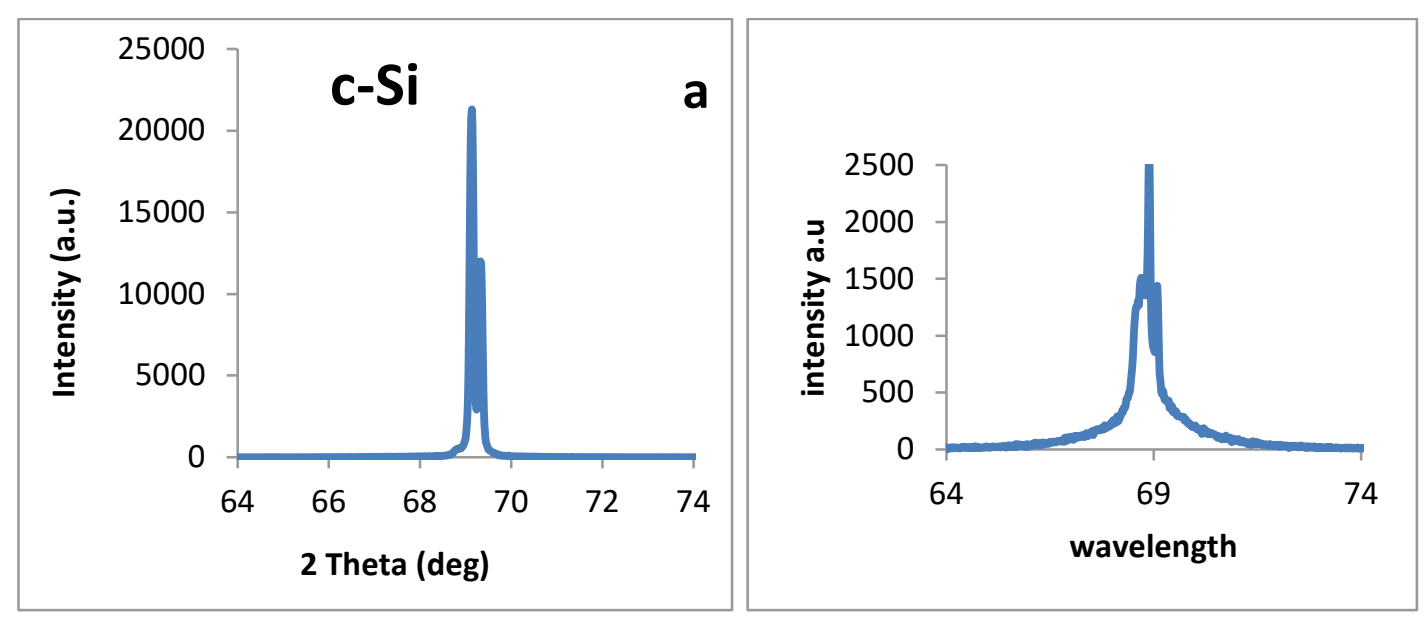

Figure 3. X-ray diffraction scheme for p-Psi

\section{Atomic force microscopy (AFM)}

In this section we present a study of some of the structural and porous properties of P-Psi. Fig. 4 shows the three-dimensional image and the (P-PSi) accumulation diagram for etching time (10min). P-Psi was measured from the AFM and was found to be about $80.63 \mathrm{~nm}$. Table (2) shows information 
on P-Psi. The surface topography of the P-Psi layer investigated by AFM shows a smooth and highly homogeneous structure

Table 2. The surface roughness values, the square root mean of the roughness square, and the particle size ratio, as measured by AFM of (P-Psi)

\begin{tabular}{|c|c|c|c|c|}
\hline $\begin{array}{c}\text { Etching Time } \\
(\mathrm{min})\end{array}$ & $\begin{array}{c}\text { Grain Size } \\
(\mathrm{nm})\end{array}$ & $\begin{array}{c}\text { Roughness } \\
\text { Average }(\mathrm{nm})\end{array}$ & $\begin{array}{c}\text { Root Mean } \\
\text { Square } \\
(\mathrm{nm})\end{array}$ & $\begin{array}{c}\text { Porosity } \\
\%\end{array}$ \\
\hline 10 & 80.63 & 5.7 & 6.58 & $67 \%$ \\
\hline
\end{tabular}

Table 2 shows the surface roughness values, the square root mean of the roughness square, and the particle size ratio, as measured by AFM of (P-Psi)
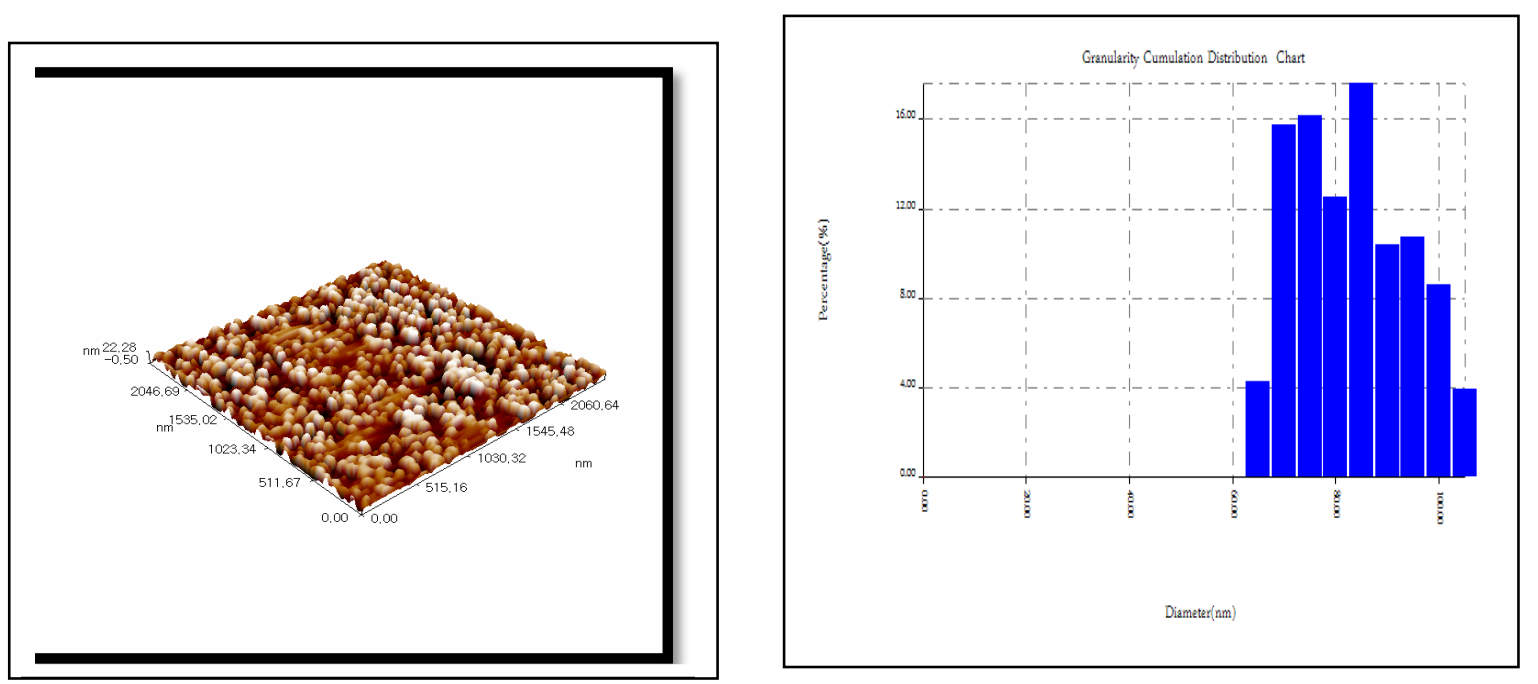

Figure 4. Results of Atomic Force Microscopy (AFM) and percentage distribution of surface particle size (P-Psi)

\section{Photoluminescence (PL) for Porous Silicon}

The properties of fluorine (PL) in porous silicon layers gave a significant boost to the study of materials due to the wide possibilities of their applications. The P (P-Psi) spectra at $\left(10 \mathrm{~mA} / \mathrm{cm}^{2}\right)$ and the time of the drilling (10min) as shown in Fig. 5 indicate an emission peak at $860 \mathrm{~nm}$ and wavelength emission at $830 \mathrm{~nm}$. (PL) at the spectral range $(1.44 \mathrm{eV})$ and can be attributed to the luster of the silicon surface 


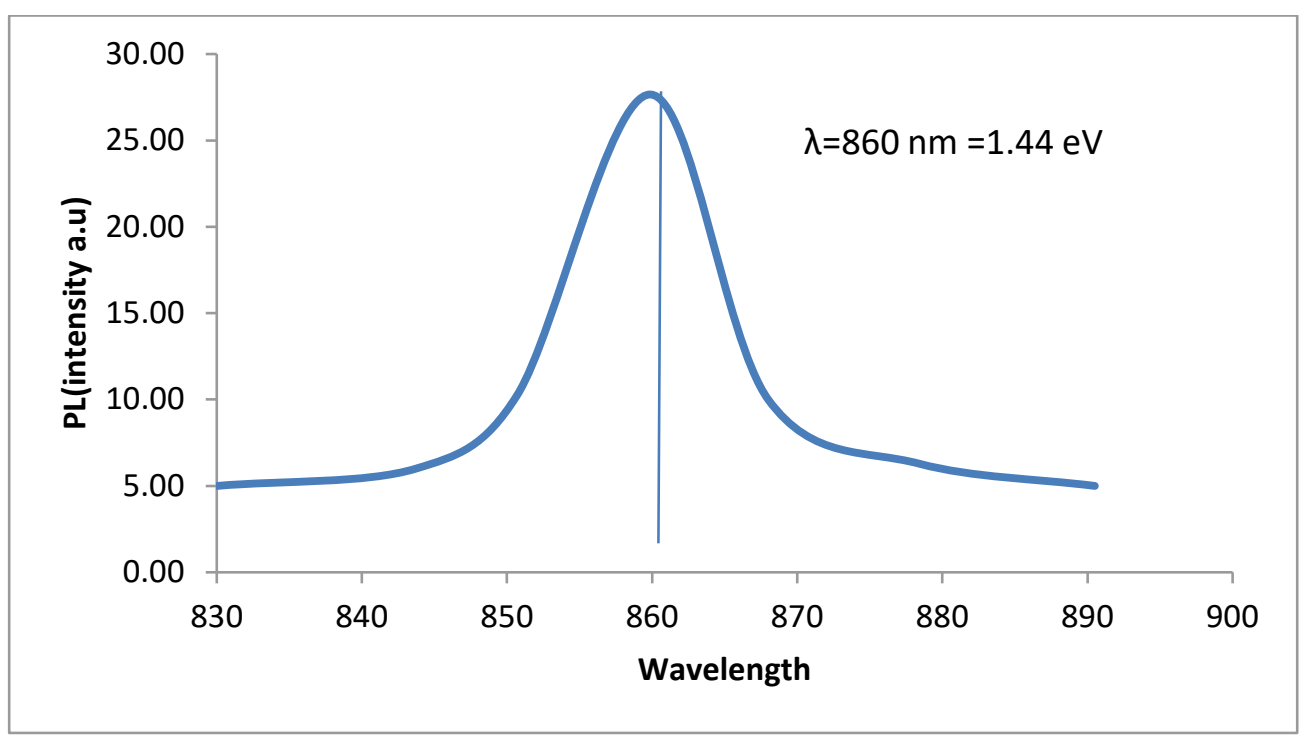

Figure 5. The properties of fluorine for (P-Psi)

\section{Conclusions}

$\mathrm{n}$ and $\mathrm{p}$ type porous silicon layer exhibits dramatic and special structure characterized by the presence of interconnected pores in a single crystal. All morphological properties of the prepared PSi layer such as surface Shape, porosity, pore diameter, pore shape, surface roughness and Root mean square (RMS), the morphological aspect of PSi layer (crystalline or amorphous), crystalline size, microstrain, lattice constant and nanocrystallite size are strongly dependent on the etching time and current density. These features of PSi have been studied by direct imaging for its structure by Optical microscopy, Atomic Force Microscopy AFM, X-ray diffraction technique (XRD).

\section{Conflict of Interest}

The authors declare that there is no conflict of interest.

\section{References}

[1] M.S. Alsalhi, Introduction to Nanotechnology, King Saud University, 2007.

[2] M. Ratner, D. Ratner, Nanotechnology Gentle Introduction, Harvard University, Brown University, 2002.

[3] A. Yousef, A. Mosleh, Manufacturing and studying the properties of a light detector, M.Sc., University of Tikrit, 2011.

[4] A. Zweck, Technological analysis, Future Technologies Division, Germany, 2004.

[5] S. Rengaraj etc., CdS microspheres composed of nanocrystals and their photocatalytic activity. Journal of Nanoscience and Nanotechnology. 11(3) (2011) 2090-2099.

[6] C. Baratto etc., Multiparametric porous silicon sensors, Sensors. 2(3) (2002) 121-126.

[7] L.T. Canham, Silicon quantum wire array fabrication by electrochemical and chemical dissolution of wafers, Applied Physics Letters. 57(10) (1990) 1046-1048.

[8] M. Megens et al., Fluorescence lifetimes and linewidths of dye in photonic crystals, Physical Review A. 59(6) (1999) 4727.

[9] L.T. Canham, Silicon quantum wire array fabrication by electrochemical and chemical dissolution of wafers. Applied Physics Letters. 57(10) (1990) 1046-1048. 
[10] S. Ossicini, L. Pavesi, F. Priolo, Light emitting silicon microphotonic, Vol. 194. Springer Science \& Business Media, 2003.

[11] P. Harrison, Q. Wells, Wires and Dots: Theoretical and Computational Physics, Wiley, New York, 2000.

[12] A. Irrera, Light emitting devices based on silicon nanostructures, Universita Degili Studidi Catania, Dottorato Diricerca in Scienza Materiali, 2003.

[13] A. Ahmed, A. Alwan, N. Alrawi, Electrical properties of porous silicon prepared by photochemical etching, Solid State Science and Technology, Proceedings of XXII Regional Conference on solid State Science and Technology, 18-21 December, 2005, Malaysia. 